\title{
Management of Subintimal Position of Kissing Stents using Re-entry Catheter with Cone Beam-Computed Tomography Image Overlaid onto Live Fluoroscopy
}

\author{
Lorenzo C Pescatori MD ${ }^{1}$, Hicham Kobeiter MD 1,2, Haytham Derbel MD', \\ Pascal Desgranges MD ${ }^{3}$ and Vania Tacher MD ${ }^{1,2,4}$ \\ 'Radiology Department, Henri Mondor Hospital, Assistance Publique-Hôpitaux de Paris, Créteil, France \\ ${ }^{2}$ Paris-Est Creteil University (UPEC), Val-de-Marne, Créteil, France \\ ${ }^{3}$ Department of Vascular Surgery, Henri Mondor Hospital, Assistance Publique-Hôpitaux de Paris, Créteil, France \\ 4INSERM U955, Team 18, Créteil, France
}

\begin{abstract}
Background: Several endovascular or surgical treatments have been proposed to treat total chronic occlusions of the iliac bifurcation. Nowadays, endovascular options are considered as a first choice because of the decreased perioperative morbidity-mortality. Nevertheless, unexpected intraoperative events may occur, such as dissection or rupture of the iliac artery. We report a case of inadvertent bilateral false-lumen kissing-stent positioning, rescued with stent extensions maneuvered using image fusion guidance.

Case presentation: A 60-year-old male patient was referred to our department because of a severe bilateral claudication, 8 months after placement of an iliac kissing stent for common iliac artery occlusion. A computed tomography angiography (CTA) was performed, showing a bilateral dissection of the aorto-iliac bifurcation at the proximal part of the stents, which were placed into the false lumen; the distal part was placed into the true lumens (TL). Lower-limb perfusion was maintained by inferior mesenteric and hypogastric arteries. Because a CTA performed before the first endovascular intervention showed no dissection of the aortic bifurcation, the flaps were probably created during previous interventions. An endovascular revision was planned. After bilateral femoral access, the proximal part of the flap was pierced with a needle-based re-entry device, deployed under three-dimensional cone-beam CT image overlay with bi-planar fluoroscopy. The lumen patency was then restored with stent extensions up to the renal ostia. The final angiography showed stent patency. No complication occurred during the intervention. The patient was dismissed the following day, with good arterial femoral pulse and no further complications. An ultrasound color Doppler performed 1 month after the intervention showed satisfactory blood flow of both iliac and femoral arteries as well as a good flow in the lower limbs.

Conclusions: The integration of modern 3D image guidance and novel endovascular devices allows for the management of adverse events using a minimally invasive approach.
\end{abstract}

Keywords: Re-entry Catheter; Subintimal Recanalization; Kissing Stent; CBCT; Fusion Imaging

Received: 4 February 2021; Accepted: 26 February 2021

\section{Corresponding author:}

Lorenzo Carlo Pescatori, Radiology Department, Henri Mondor Hospital, Assistance Publique-Hôpitaux de Paris, Créteil, France.

Email: Iorenzo.carlo.pescatori@gmail.com

(c) 2021 CC BY 4.0 - in cooperation with Depts. of Cardiothoracic/ Vascular Surgery, General Surgery and Anesthesia, Örebro University Hospital and Örebro University, Sweden

\section{INTRODUCTION}

Atherosclerotic occlusion of the aorto-iliac bifurcation is a common cause of claudication and/or critical limb ischemia, with a prevalence ranging between 3 and $10 \%$ [1]. Different treatment options exist to restore blood flow to the lower limb (i.e. surgical or endovascular), but unexpected intraoperative events may occur, such as dissection or rupture of the iliac artery [1,2]. Herein, we 

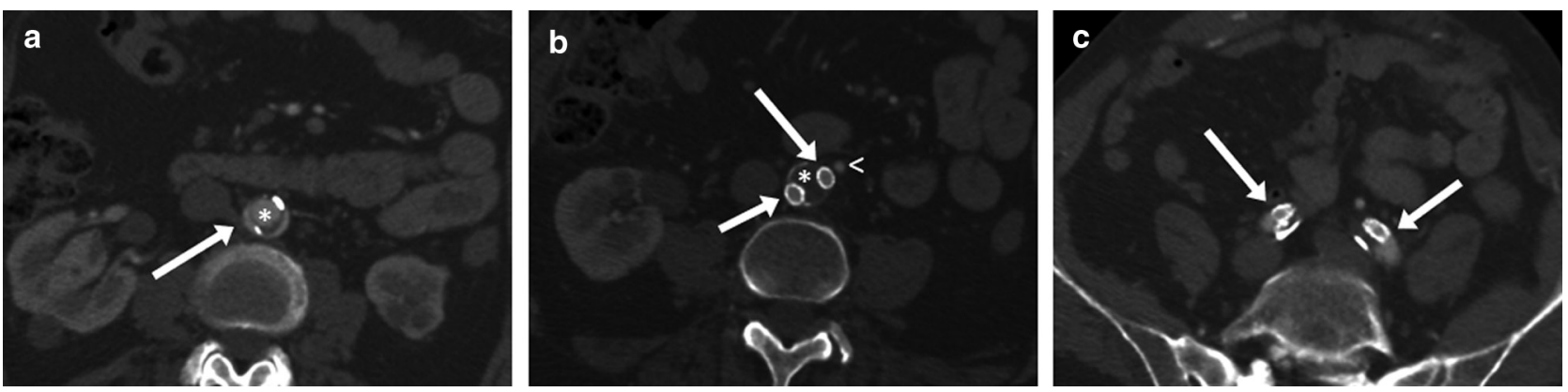

Figure 1 (a) Axial view of the aortic dissection over the proximal part of the iliac stents, with both the true lumen (asterisk) and the false lumen (arrow) still patent. (b) Dissection of the distal part of the aorta, showing thrombosis of the true lumen (asterisk) and patency of the two iliac stents (arrows). Axial view of a hypertrophic inferior mesenteric artery is shown (arrowhead). (c) Distal part of the iliac stents, showing patency of the lumen and the native iliac arteries (arrows).
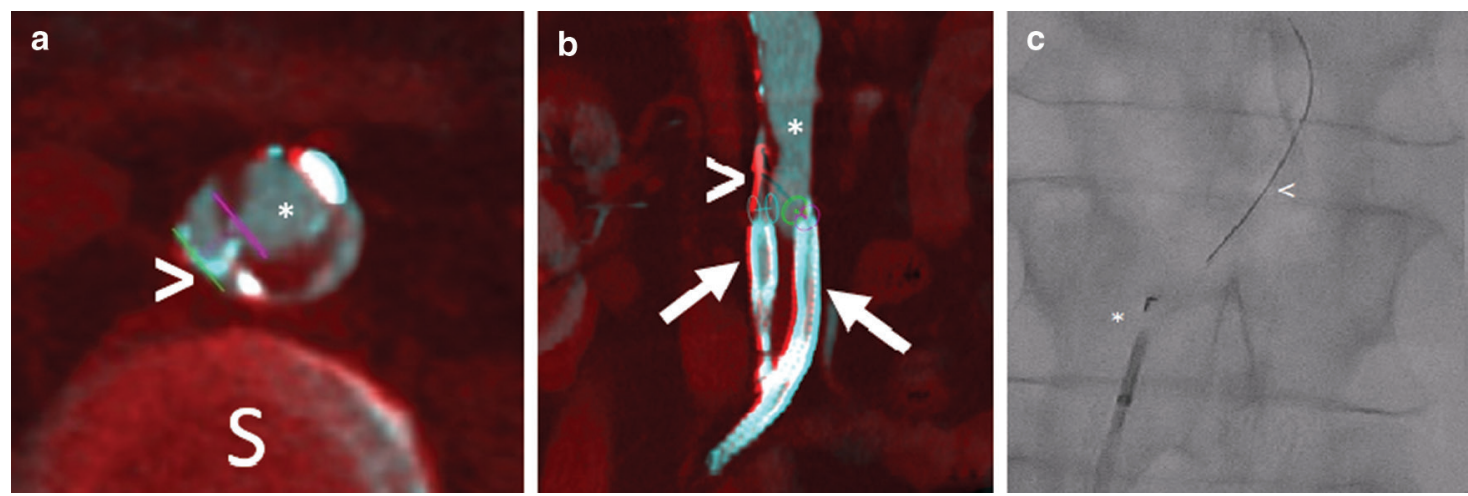

Figure 2 Axial (a) and coronal (b) cone beam computed tomography (CBCT) merged with pre-operative computed tomography angiography (CTA) showing the stents (arrows) in the false lumen, a virtual needle trajectory drawn between the true (asterisk) and false lumen (purple, green and light blue circles). The tip of a 0.014 " guidewire is stuck at the top of the false lumen (arrowhead). (c) Outback re-entry catheter with the distal part in the false lumen (asterisk) and the opened sharp tip piercing the intimal flap. The tip of the guidewire (arrowhead) is passed through the flap into the true lumen.

report a case of an inadvertent bilateral false-lumen kissing-stent positioning into the aorto-iliac bifurcation, rescued with stent extensions maneuvered using image fusion guidance.

\section{Ethical Approval and Informed Consent}

Ethical approval is not required for retrospective works, at our Institution. Written informed consent was obtained from the patient.

\section{CASE REPORT}

A 60-year-old male patient was referred to our institution at the beginning of April 2019 because of a severe claudication of both legs, occurring after $50 \mathrm{~m}$ of walking. Because he had been treated with an iliac kissing stent for common iliac artery occlusion 8 months prior, an in-stent restenosis was suspected.

Computed tomography angiography (CTA) of the abdominal aorta and lower limbs was performed, showing dissection of the aorto-iliac bifurcation at the proximal portions of the stents that were placed into the false lumen; in contrast, the distal portions of both stents were shown situated in the true lumens (TL). Despite focal obliteration of the TL, lower-limb perfusion was maintained via inferior mesenteric and hypogastric arteries (Figure 1). Because CTA performed before the first endovascular intervention showed no dissection of the aortic bifurcation, we hypothesized that the flaps were created during the previous intervention and the stents were inadvertently deployed into the false lumen. Thus, an endovascular revision was proposed.

\section{Endovascular Management}

The intervention was carried out in an interventional radiology suite equipped with a biplanar flat panel, a cone beam CT (CBCT) (Azurion 7 B20/15, Philips Healthcare, Best, The Netherlands) and XperCT 3D reconstruction software (Philips Healthcare).

A 6-Fr, 13-cm-long sheath was introduced into the femoral common artery, on both sides. Then, 4-Fr catheters (Bernstein, Cordis, Milpitas, CA) were directed 

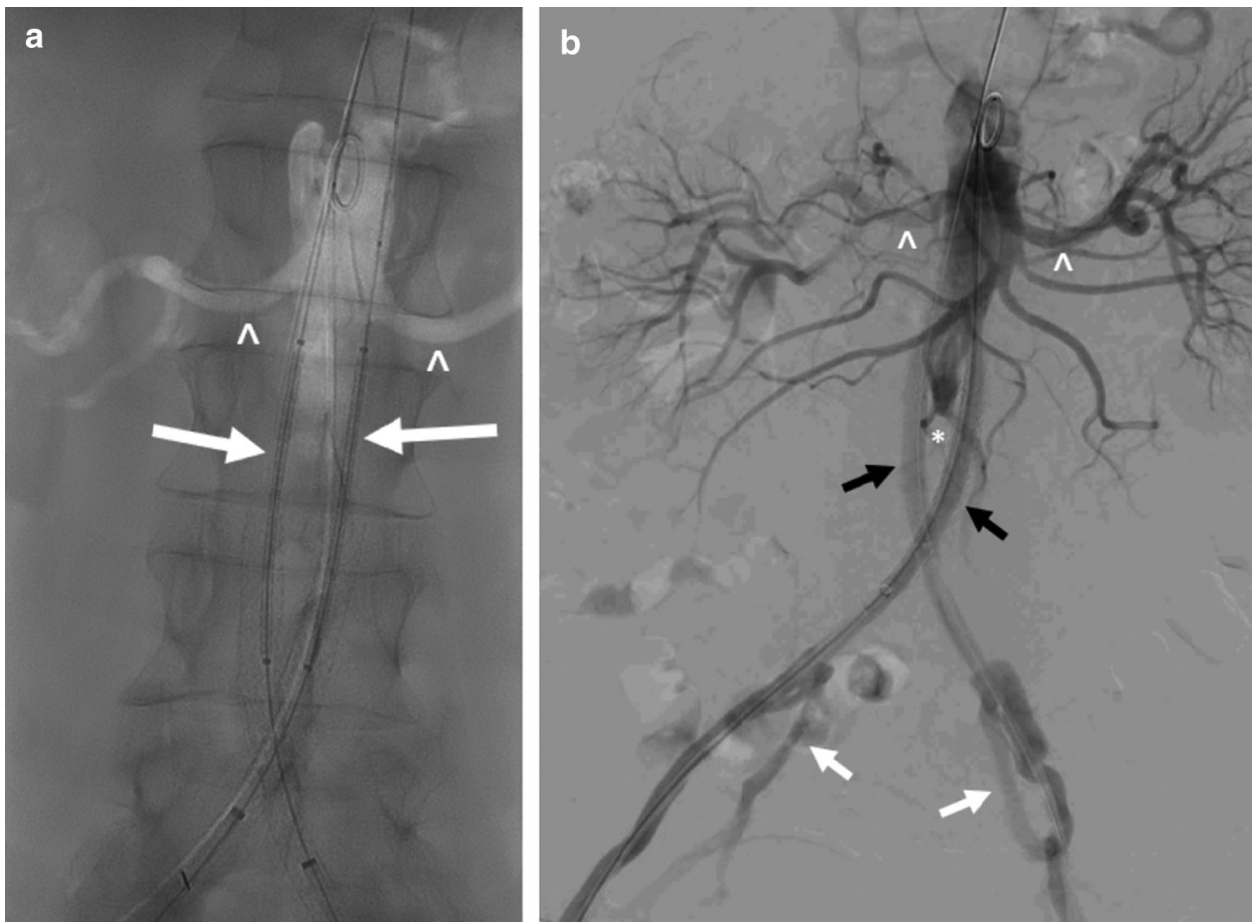

Figure 3 (a) Once the guidewires were placed into the true lumen on both sides, two balloonexpandable covered stents (arrows) were deployed in the abdominal aorta, with the proximal part just below the renal arteries (arrowheads) and the distal part overlapping the stents previously deployed. (b) Final angiography showing restored aorto-iliac flow through the stents (black arrows), with thrombosis of the dissected distal aorta (asterisk), patent renal (arrowheads) and hypogastric (white arrows) arteries.

into the top of the iliac stents. A CBCT was performed to merge images with those of the pre-treatment CTA, as described elsewhere [3].

After review of the multiplanar overlaid images, ideal virtual re-entry needle trajectories were marked on the workstation. C-arm inclinations corresponding to entry point and needle progression views were defined for each trajectory; virtual needle trajectories were merged onto fluoroscopy images to create 3D images providing guidance for the re-entry devices (Figure 2).

A re-entry catheter (Outback Elite Re-Entry Catheter, Cordis, Miami, FL) was passed through the iliac stents, and the tip of the device was deployed according to the trajectory provided by the 3D images (Figure 2) and followed by means of bi-planar fluoroscopy in order to keep control of the re-entry point and the needle progression, simultaneously.

The flap was perforated on the first attempt, on both sides, and a steel guidewire (0.014" Spartacore, Abbott Vascular, Abbott Park, IL) was placed in the TL of the aorta. Two glide catheters were inserted, and a control angiography was performed to confirm the position into the TL.

Subsequently, two 7-Fr, 55-cm-long sheaths (Cook Medical, Bloomington, Ind) were inserted and two 7-mm-wide, 59-mm-long Advanta V12 balloon expandable covered stents (Atrium Europe B.V, Mijdrecht, The Netherlands) were deployed, with $1 \mathrm{~cm}$ overlapping the previous stents and the remaining part inserted in the TL of the aorta, thus restoring direct connection between the aorta and iliac arteries (Figure 3). A final angiography showed stent patency and no complications occurred during the intervention (Figure 3).

At the end of the procedure, the femoral accesses were closed using 8-Fr Angioseals (Terumo, Tokyo, Japan). The patient was dismissed the following day, with a good arterial femoral pulse and no further complications. An ultrasound colour Doppler performed 1 month after the intervention showed satisfactory blood flow of both iliac and femoral arteries as well as a good flow in the lower limbs.

\section{CONCLUSION}

We have reported the endovascular management of a complication that occurred during stenting of the aortoiliac bifurcation. Dissection, especially when bilateral, can cause distal embolization, worsening claudication or loss of hypogastric vascularization, with subsequent gluteal necrosis, ischemic colitis, impotence and spinal ischemia [4]. Typically, the operator should suspect an intraprocedural iliac dissection when the pressure gradient between the pre- and post-stenotic tract is lost. During the operation, the suspicion can be confirmed in several ways, although the most reliable seems to be an evaluation 
of either the artery wall using intravascular ultrasound or the vascular network using an injected CBCT, allowing to check for both the position of the catheter and the patency of the artery [5].

Days after the procedure, the patient should undergo a duplex ultrasound to explore the flow restoration. In the case of neither flow restoration nor clinical improvement, a CTA should be performed to search for the reasons behind the procedure's inefficacy.

In the presented case, dissection was limited to the common iliac arteries and did not cause an acute lowerlimb ischemia as the patient suffered from chronic claudication due to bilateral iliac artery occlusion and he already had a hypertrophic collateral vascular supply to the pelvis and lower limbs.

Nowadays, the kissing-stent technique is the first-line treatment for proximal common iliac artery stenosis, because of its lower complication rate relative to open surgery [1]. Nevertheless, iliac manipulation remains challenging, because complications, although rare, can lead to major consequences [4]. Different rescue options have been discussed, and an endovascular approach has been decided as first choice, in order to keep surgery as a second option, in case of failure. Thus, the intervention was carried out using a combination of tools and techniques, well known but never used together so far.

Aortic fenestration was described in 1990 for the first time [6] and, since then, many techniques have been proposed for this purpose. The re-entry catheter with a needle tip facilitates passage through the endothelial flap and allows direct cannulation of TL [7]. The maximal extension of the tip is $10 \mathrm{~mm}$, which was enough in this case as the TL was narrowed and compressed between two flaps on both sides.

As shown, the possibility to acquire 3D images in the angiography suite and to overlay them with pictures from fluoroscopy, allows safe and precise planification of challenging interventions that, otherwise, would require multiple controls and long X-ray exposure [8].

In this case, the availability of a biplanar machine may have reduced the intervention time, allowing simultaneous control of entry point and progression trajectory, unfeasible with a single panel.

In conclusion, the opportunity to integrate modern 3D image guidance and the high-performing endovascular devices allowed safe and rapid management of adverse events using a minimally invasive approach.

\section{Ethics Statement}

(1) All the authors mentioned in the manuscript have agreed to authorship, read and approved the manuscript, and given consent for submission and subsequent publication of the manuscript.

(2) The authors declare that they have read and abided by the JEVTM statement of ethical standards including rules of informed consent and ethical committee approval as stated in the article.

\section{Conflicts of Interest}

The authors of this manuscript declare no relationships with any companies whose products or services may be related to the subject matter of the article.

\section{Funding}

The authors state that this work has not received any funding.

\section{Author Contributions}

HK, HD, PD, and VT conceived the presented case. LCP wrote the manuscript. VT and HK supervised the project. All authors discussed the results and contributed to the final manuscript.

\section{REFERENCES}

[1] Groot Jebbink E, Holewijn S, Slump CH, Lardenoije JW, Reijnen MMPJ. Systematic review of results of kissing stents in the treatment of aortoiliac occlusive disease. Ann Vasc Surg. 2017;42:328-36. doi:10.1016/j.avsg. 2017.01.009.

[2] Vacirca A, Faggioli G, Pini R, et al. The outcome of technical intraoperative complications occurring in standard aortic endovascular repair. Ann Vasc Surg 2019;56:15362. doi:10.1016/j.avsg.2018.08.092.

[3] Tacher V, Lin M, Desgranges P, et al. Image guidance for endovascular repair of complex aortic aneurysms: comparison of two-dimensional and three-dimensional angiography and image fusion. J Vasc Interv Radiol. 2013;24:1698-706. doi:10.1016/j.jvir.2013.07. 016.

[4] Stone PA, Campbell JE, Hass SM, Jain A, Kazmi H. Kissing iliac artery stent technique for salvage of hypogastric artery occlusion secondary to iliac artery dissection during endovascular aneurysm repair. Vasc Endovasc Surg. 2011;45:174-7.doi:10.1177/1538574410377240.

[5] Törnqvist P, Dias N, Sonesson B, Kristmundsson T, Resch T. Utility of intra-operative cone beam computed tomography in endovascular treatment of aorto-iliac occlusive disease. Eur J Vasc Endovasc Surg. 2016;51:358-63. doi:10.1016/j.ejvs.2015.09.019.

[6] Williams DM, Brothers TE, Messina LM. Relief of mesenteric ischemia in type III aortic dissection with percutaneous fenestration of the aortic septum. Radiology. 1990;174:450-2. doi:10.1148/radiology.174.2. 2136956.

[7] Varu VN, Lee GK, Chang S, Lee JT. Reentry device aided endovascular aneurysm repair in patients with abdominal aortic aneurysm and unilateral iliac artery occlusion. Ann Vasc Surg. 2014;28:1800.e1-7. doi:10.1016/j.avsg. 2014.05.008.

[8] Tacher V, Petit A, Derbel H, et al. Three-dimensional image fusion guidance for transjugular intrahepatic portosystemic shunt placement. Cardiovasc Intervent Radiol. 2017;40:1732-1739. doi:10.1007/s00270-0171699-9. 